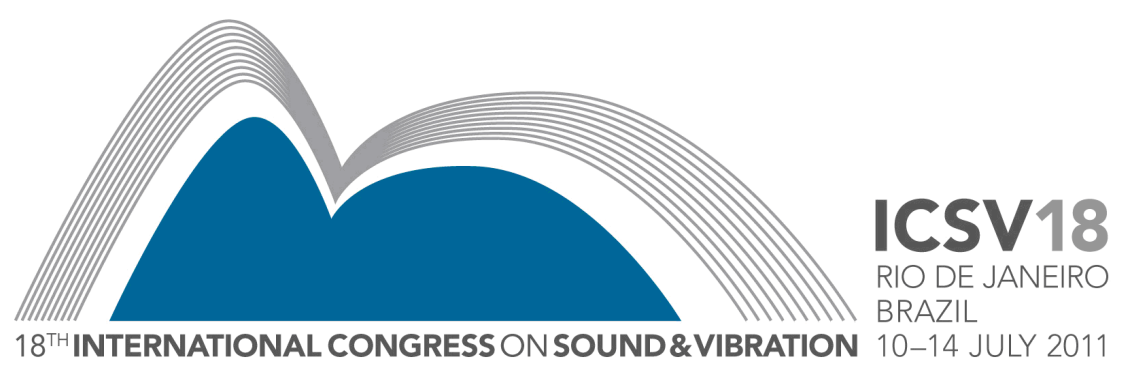

\title{
ACOUSTIC SOURCE DATA FOR MEDIUM SPEED IC-ENGINES
}

\author{
Antti Hynninen and Raimo Turunen \\ VTT Technical Research Centre of Finland, P.O. Box 1000 FI-02044 VTT, Finland, \\ e-mail: antti.hynninen@vtt.fi \\ Mats Åbom and Hans Bodén \\ KTH CCGEx, Marcus Wallenberg Laboratory, SE-10044 Stockholm, Sweden
}

\begin{abstract}
Knowledge of the acoustic source characteristics of internal combustion engines (IC-engines) is of great importance when designing the exhaust duct system and its components to withstand the resulting dynamic loads and to reduce the exhaust noise emission. Number of studies has been published earlier on the low frequency in-duct exhaust noise of high speed engines. The goal of the present study is to investigate the medium speed IC-engine acoustic source characteristics numerically and experimentally not only in the low frequency - plane wave range but also in the high frequency range. The low frequency acoustic source characteristics were predicted by simulating the acoustic multi-load measurements using a one-dimensional process simulation code. The engine model used in the one-dimensional process simulations was validated with measurements. In this study, it is shown that the low frequency in-duct exhaust noise of a medium speed IC-engine can be predicted quite accurately by using a onedimensional process simulation code. The high frequency source data is estimated by averaging the measured acoustic pressures with different methods. According to this study, using the simple cross spectra averaging method instead of two microphone method to estimate the induct downstream acoustic power of medium speed IC-engine exhaust noise seems promising. The simulation of the high frequency exhaust noise is beyond this study.
\end{abstract}

\section{Introduction}

Noise legislations and the shorter product development cycles increase the need for more accurate predictions of the noise generation of machines in the early design phase i.e. before prototyping. To reduce the need for trial and error iterations in the product development process, the measuring methods must be as simple and robust as possible.

Medium speed IC-engines are used in large electrical generators, ship propulsion systems and mechanical drive applications such as large compressors and pumps. The rotation speed of a medium speed IC-engine is typically from 300 to $1000 \mathrm{rpm}$ whereas high-speed IC-engines used to power e.g. trucks, buses and cars usually run at $1000 \mathrm{rpm}$ or more.

\subsection{Frequency and time-domain analysis}

For acoustic analysis of intake and exhaust systems often linear models are preferred compared to non-linear ones because of computational efficiency and accuracy of acoustic loss models. Using 
linear models frequency-domain analysis is a convenient way to design exhaust system and its components, e.g. mufflers and silencers. The acoustic load-independent source data of an IC-engine is needed as an input in the frequency-domain simulations.

The gas dynamics of an IC-engine can essentially be described by a set of coupled nonlinear equations for conservation of mass, momentum and energy. The most widely used method to solve these nonlinear equations in time-domain is to use a finite volume or finite difference method. The methods and examples concerning IC-engines are explained in-depth e.g. in the book by Munjal [1] and in the books by Winterbone and Pearson [2,3]. The governing equations are often simplified by treating the variables such as pressure, density, velocity and temperature as constant over the duct cross-section. One-dimensional commercial process simulation software such as AVL-Boost, Ricardo-Wave and GT-Power are the most widely used within the automotive industry. These codes are mainly used to predict IC-engine performance quantities such as volumetric efficiency, torque and power. In addition, the codes provide the pressure and flow velocities at the intake and exhaust system.

There are also so called hybrid modelling approaches where the time-domain and the frequencydomain methods are combined. Several hybrid approaches has been reviewed in the state of the art review of the acoustic source characterization of an IC-engine by Munjal [4] and in the paper by Albertson et al. [5]. The hybrid methods studied in these papers were found to be computationally expensive and rather complex.

In this study the medium speed IC-engine exhaust duct acoustics is studied by using linear frequency-domain methods.

\section{Methods}

The low frequency - plane wave range acoustic source characteristics of a medium speed ICengine were obtained by simulating the acoustic multi-load method measurements. A commercial one-dimensional process simulation software GT-Power [6] was used to solve the nonlinear set of partial differential equations describing the gas dynamics of the IC-engine. The simulation model of the IC-engine was validated with engine performance and cylinder pressure measurements. The measured acoustic pressure $\hat{p}$ and acoustic load impedance $\hat{\zeta}_{L}$ of the exhaust piping was used to estimate the accuracy of the simulated low frequency - plane wave range source data, i.e. source pressure $\hat{p}_{s}$ and source impedance $\hat{\zeta}_{s}$.

The high frequency range was included in the acoustic power estimation using spatial averaging methods. The source data was estimated by averaging the measured auto- and cross-spectra and assuming negligible reflections and a semi-diffuse field. In the high frequency range measurements, the acoustic pressures were measured from several points in the same measurement section of the exhaust duct. The sound powers achieved with different methods were compared.

\subsection{Determining the source characteristics}

A review on the experimental methods for determining the source data of fluid machines is presented in the paper by Bodén and Åbom [7]. The methods can be divided into direct and indirect methods.

The source impedance of the IC-engine can be measured directly by using an external source that would produce a stronger sound field than the noise produced by the engine. As stated in the papers by Gupta and Munjal [8] and Munjal [4], acoustically strong enough sources are practically impossible to construct in order to study sound from an IC-engine.

The source characteristics of the IC-engine can be measured indirectly via a multi-load procedure i.e. by applying $m$ known acoustic load impedances $\hat{\zeta}_{L m}$ to the system and measuring the corresponding acoustic pressures $\hat{p}_{0 \mathrm{Lm}}$. That kind of indirect method does not need any secondary 
acoustic source. Since there are only two unknowns, two measurements should be sufficient, i.e. $m=1 \ldots 2$. This leads to the two-load method.

In order to reduce the influence of the measurement errors and deviation from linearity more than two loads can be used. This procedure leads to an over-determined problem. The source characterization with different multi-load methods is described in detail in the paper by Bodén [9].

To get reliable results with the multi-load method measurements several different duct constructions are needed. Because of the size of the medium speed IC-engine and its exhaust system, measuring the source characteristics with the multi-load method is too time consuming and practically impossible.

\subsubsection{Multi-load method simulations}

The source characteristics of an IC-engine can be obtained by simulation using the multi-load method. That kind of approach has been used earlier to study high-speed engines. The acoustic source data of a six cylinder turbo-charged truck diesel engine exhaust system has been determined in the papers by Bodén et al. [10] and Fairbrother et al. [11]. The source data of a four-cylinder passenger car petrol engine exhaust system and six cylinder truck diesel intake system were studied in the paper by Bodén [12]. The acoustic source data of a six cylinder passenger car petrol engine intake system was studied in the paper and thesis by Knutsson [13, 14].

The obvious difference in this study compared to the earlier ones is the size of the structure in focus; e.g. the typical diameter of the automotive exhaust pipe is approximately $50 \mathrm{~mm}$ whereas the diameter of the medium speed IC-engine exhaust piping typically varies between 0.4 and $1.4 \mathrm{~m}$. The diameter of the exhaust duct is inversely proportional to the cut-on frequency of the first non-plane wave mode, e.g. in temperature of $500{ }^{\circ} \mathrm{C}$ the cut-on frequency in typical automotive exhaust pipe of $\varnothing=50 \mathrm{~mm}$ is $5860 \mathrm{~Hz}$, whereas in the medium speed IC-engine exhaust duct of $\varnothing=500 \mathrm{~mm}$ the cut-on frequency is $586 \mathrm{~Hz}$.

Also the operating conditions of a medium speed IC-engine are more constant than in passenger cars or trucks.

\subsection{Acoustic power estimation using wave decomposition}

According to Neise et al. [15] the time averaged downstream acoustic power can be estimated by summing over the cut-on modes as

$$
\bar{W}_{+}=\frac{1}{Z_{0}} \sum_{n=0}^{N} \varepsilon_{n} \tilde{p}_{n+}^{2},
$$

where $Z_{0}=\rho c / A$ is the characteristic impedance for a propagating plane wave in a duct with cross sectional area $A$ filled with gas of density $\rho, c$ is the speed of sound in the gas mixture, $\varepsilon_{n}$ is a weighting factor for each mode, $\tilde{p}_{n}$ is the root mean square of the acoustic pressure, $n$ is the mode number and + denotes propagation to downstream direction.

To obtain downstream acoustic power in the plane wave range $\bar{W}_{0+}$, the so called wave decomposition was done by using the two microphone method presented e.g. by Chung and Blaser [16]. The acoustical load impedance $\hat{\zeta}_{L}$ of the exhaust piping was derived according to the wave decomposition formulation presented by Bodén and Åbom [17].

If we use simulated acoustic source pressure $\hat{p}_{s}$ and simulated acoustic source impedance $\hat{\zeta}_{s}$ together with the measured load impedance $\hat{\zeta}_{L}$ we get the acoustic "simulated" pressure as

$$
\hat{p}_{0}^{\prime}=\frac{\hat{p}_{s} \hat{\zeta}_{L}}{\hat{\zeta}_{s}+\hat{\zeta}_{L}} .
$$

Using this we get the "simulated" downstream acoustic power in the plane wave range $\bar{W}_{0+}^{\prime}$. 


\subsection{Acoustic power estimation using spatial averaging}

Assuming the reflection coefficients to be negligible, the sum of downstream modal acoustic pressures in Eq. (1) can be estimated as

$$
\sum_{n=0}^{N} \varepsilon_{n} \tilde{p}_{n+}^{2}=\eta\left\langle\tilde{p}^{2}\right\rangle,
$$

where $\eta$ is the frequency range weighting factor and $\left\langle\tilde{p}^{2}\right\rangle$ is the spatial average of the squared rmspressures. If we assume a semi-diffuse field, the frequency range weighting factor $\eta=1$ for the plane wave range and $\eta=1 / 2$ after the first cut-on frequency as stated in the paper by Joseph et al. [18].

Finally using Eq. (1), the downstream acoustic power can be estimated with spatial averaging of the acoustic pressures as

$$
\bar{W}_{S+}=\frac{\eta\left\langle\tilde{p}^{2}\right\rangle}{Z_{0}}
$$

where subscript $S$ denotes for spatial averaging method.

\subsection{Acoustic power comparisons}

Comparing the sound powers $\bar{W}_{0+}$ and $\bar{W}_{0+}^{\prime}$ we can study the accuracy of the simulated source characteristics in the low frequency, plane wave range.

Comparing the sound powers achieved with auto and cross spectra $\bar{W}_{S a+}$ and $\bar{W}_{S c+}$ with sound power achieved with two microphone method measurements $\bar{W}_{0+}$, we can estimate the accuracy of the averaging methods in the low frequency, plane wave range.

Finally, by comparing the sound powers achieved with auto and cross spectra $\bar{W}_{S a+}$ and $\bar{W}_{S c+}$, the significance of the low and high frequency range can be estimated.

\section{The studied IC-engine}

The test engine studied is a Wärtsilä Vasa 4R32. The inline four-cylinder engine operates with constant speed of $750 \mathrm{rpm}$, producing $1640 \mathrm{~kW}$ power. The cylinder bore is $320 \mathrm{~mm}$ and stroke 350 $\mathrm{mm}$. The engine weights 20.3 tons (with liquids, but without flywheel). The test engine presented in Fig. 1 is located in the VTT engine laboratory in Otaniemi, Espoo Finland.

\section{Measurements}

To derive the downstream acoustic powers in the plane-wave range $\bar{W}_{0+}$ and $\bar{W}_{0+}^{\prime}$, the acoustic load impedance of the exhaust piping $\hat{\zeta}_{L}$ as well as the downstream acoustic pressures in the exhaust pipe $\hat{p}_{0+}$ were measured using two-microphone method.

The pressure was measured using six measurement points in the exhaust pipe after the turbocharger. Four pressure transducers were used in the first measurement section. In this measurement section, the four pressure transducers were mounted evenly around the pipe (measurement points 1 to 4 in Fig. 2). In the other sections, sections 2 and 3, only one pressure transducer was used at each section (measurement points 5 and 6 in Fig. 2). The measurement points and measurement sections are presented in Fig. 2.

\subsection{Downstream acoustic power estimations}

The downstream acoustic power in the plane-wave range $\bar{W}_{0+}$ as well as the sound powers derived with auto and cross spectra $\bar{W}_{S a+}$ and $\bar{W}_{S c+}$ are presented in Fig. 3. Using the averaging with auto spectra the measured pressures from measurement points 1, 5 and 6 were used for the low 


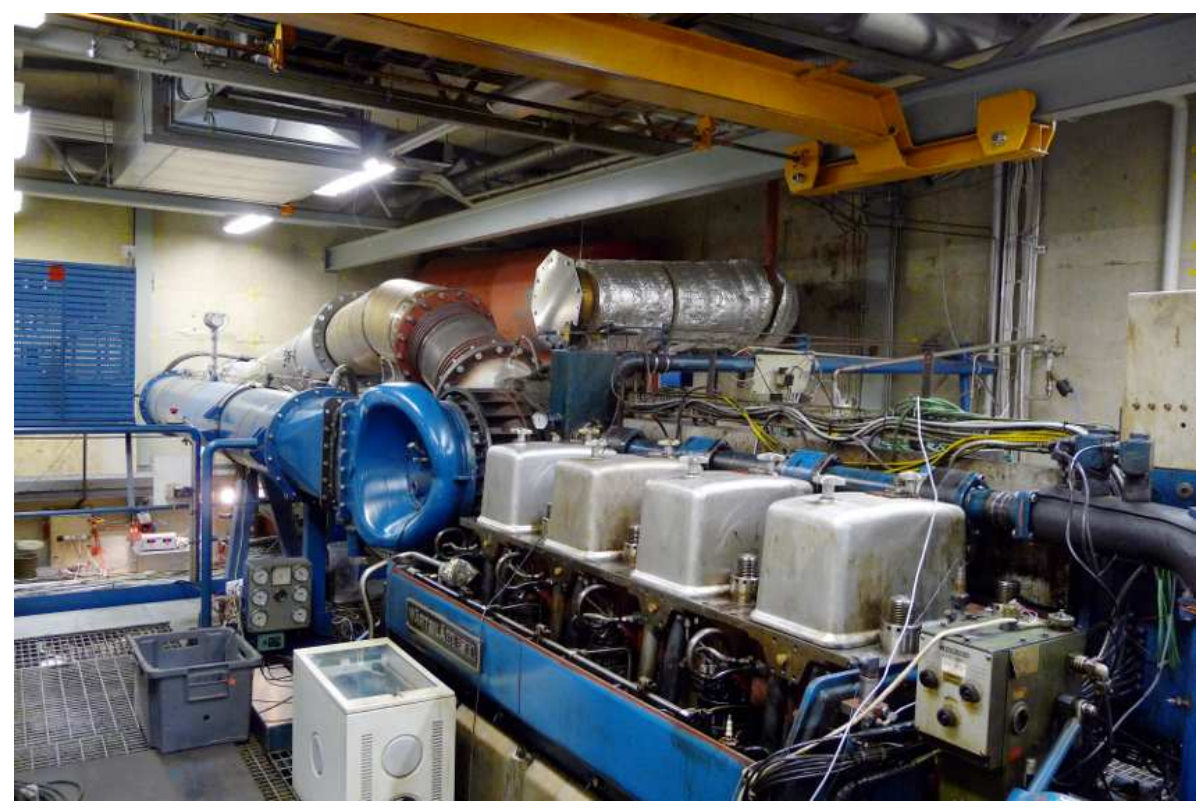

Figure 1. The test engine, Wärtsilä Vasa 4R32 in VTT engine laboratory.

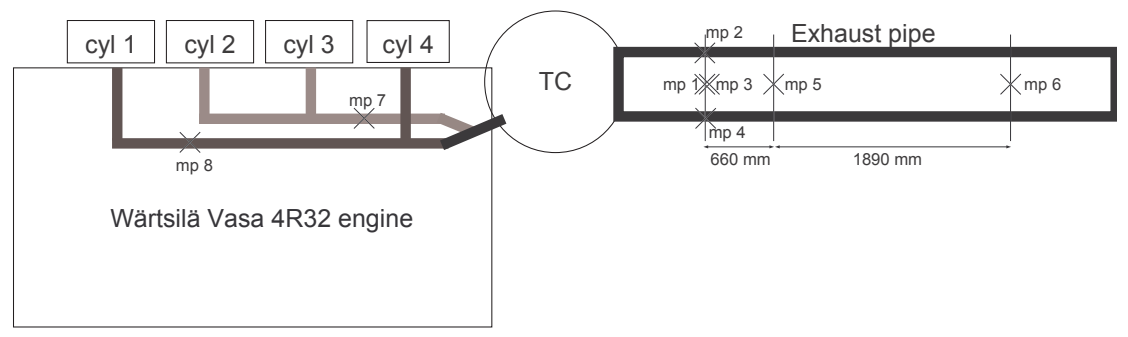

Figure 2. The measurement point locations for pressure transducers in the exhaust manifold and exhaust pipe.

frequency range and measurement points 1 to 6 for frequency range from the first non-plane wave cuton frequency onwards. Averaging with cross spectra, the measured cross spectrum from measurement point 1 to 2,1 to 5 and 1 to 6 were used for the low frequency range and all the measured cross spectra were used for frequency range from the first non-plane wave cut-on frequency onwards.

\section{Simulations}

In this study a validated engine model was used in the acoustical multi-load method simulations to determine the acoustic source data of a four-cylinder medium speed IC-engine exhaust system. By using simulated acoustic source data together with the measured acoustic load, the "simulated" downstream acoustic power in the plane wave range $\bar{W}_{0+}^{\prime}$ was derived.

\subsection{Source data simulations}

The acoustical loads needed in the multi-load method were constructed by adding side branches to the exhaust piping model after the turbo charger. In the simulation a total of ten different acoustical loads were used. The length of a side branch pipe was varied between approximately 0.8 and $11 \mathrm{~m}$.

\subsection{Downstream acoustic power simulations}

The measured and "simulated" induct downstream sound powers $\bar{W}_{0+}$ and $\bar{W}_{0+}^{\prime}$ for the low frequency - plane wave range are presented in Fig. 4. 


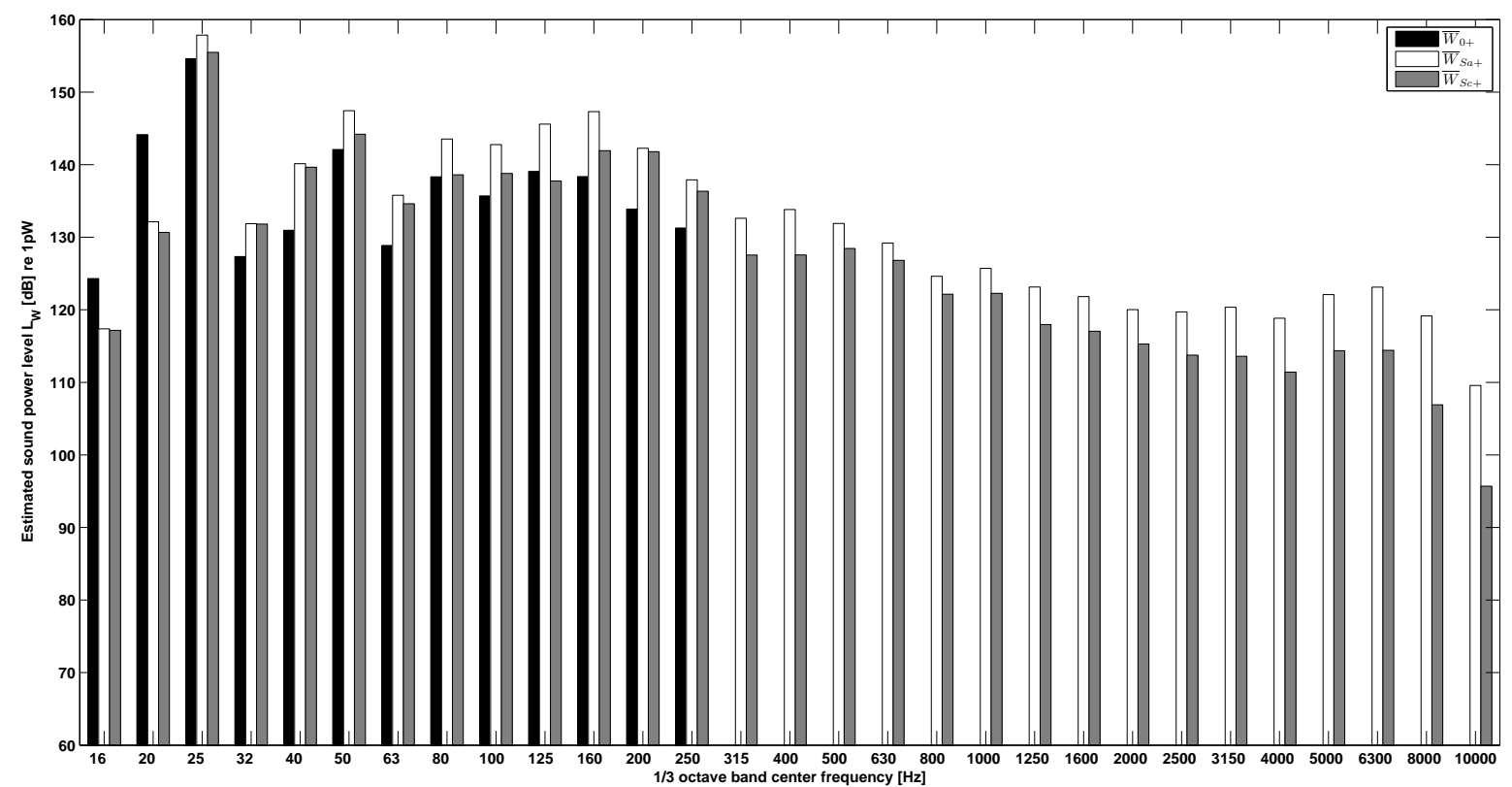

Figure 3. The downstream acoustic power levels determined at the full engine load. The plane-wave range part $\bar{W}_{0+}$ based on wave decomposition, $\bar{W}_{S a+}$ and $\bar{W}_{S c+}$ achieved by averaging the auto- and cross spectra.

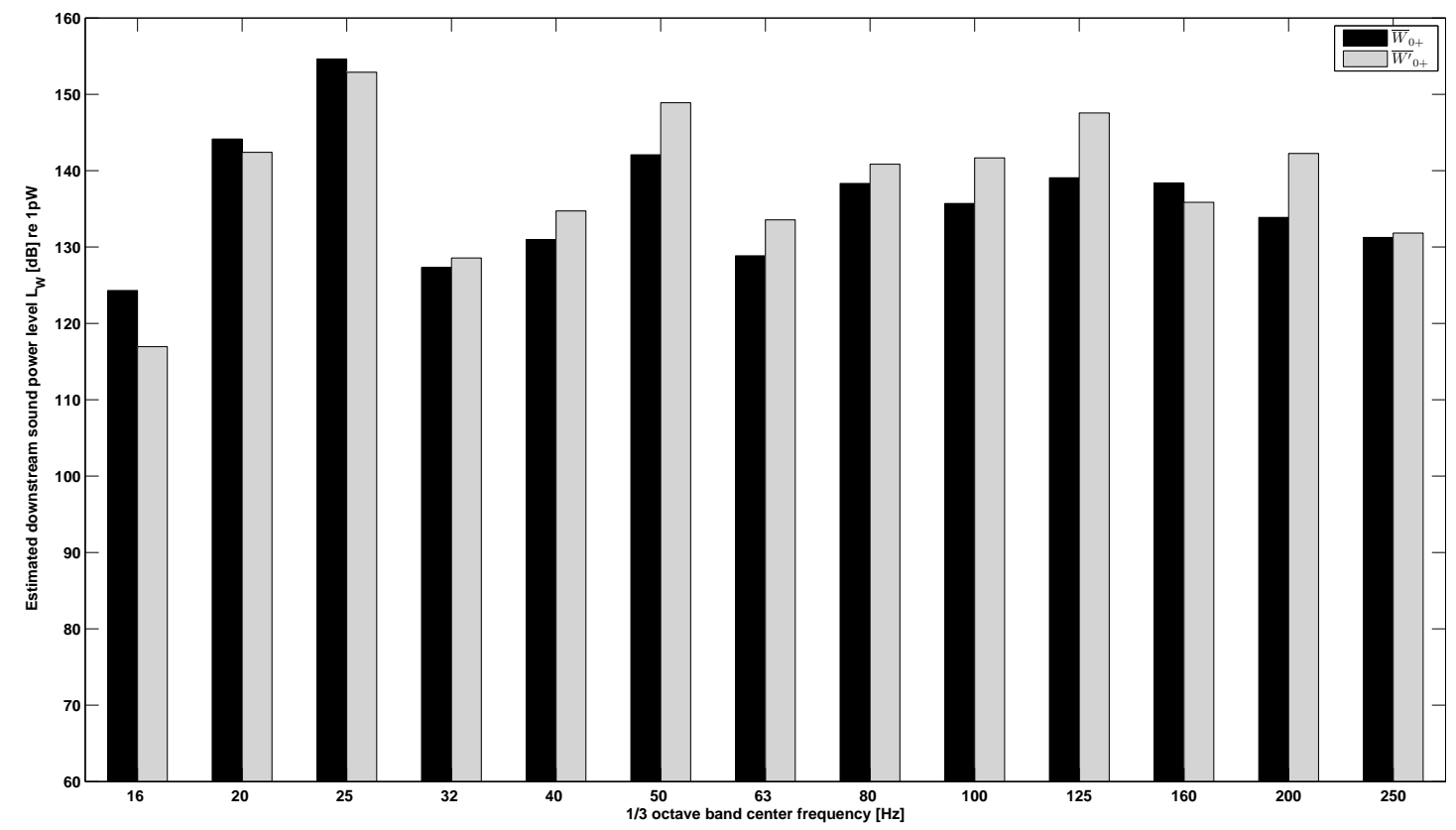

Figure 4. The measured induct sound power level $\bar{W}_{0+}$ and simulated induct sound power level $\bar{W}_{0+}^{\prime}$ in the low frequency range at the full engine load. 


\section{Discussion}

Most of the acoustic energy of the IC-engine exhaust system is dominated by the first engine cycle harmonic, i.e. the $2^{\text {nd }}$ crankshaft rotation order of a 4-cylinder four stroke engine. This can be clearly seen from the downstream acoustic power data in Fig. 3, where the bar of the 1/3 octave band of center frequency at $25 \mathrm{~Hz}$ is largest. Estimating the downstream acoustic power by using the measured acoustic pressure auto spectra gives total downstream sound power level of $159.3 \mathrm{~dB}$, discarding the non plane wave part the total sound power level is $159.2 \mathrm{~dB}$. By using the measured cross spectra the total downstream sound power level is $156.6 \mathrm{~dB}$ and $156.5 \mathrm{~dB}$ when the non plane wave part is discarded. Estimating the downstream acoustic power by using the measured acoustic pressure cross spectra gives fairly similar results as the sound power estimation based on wave decomposition at the main engine cycle harmonics, i.e. the difference of the downstream sound power level was $0.9 \mathrm{~dB}$ and $2.1 \mathrm{~dB}$ at the octave bands of center frequencies at $25 \mathrm{~Hz}$ and $50 \mathrm{~Hz}$ respectively. As a conclusion the effect of the non plane wave range sound power can be considered minor to the total sound power level.

As can be seen from the sound power level Fig. 4, the induct downstream sound power in the exhaust piping is dominated by the first engine cycle harmonic. Within this $1 / 3$ octave band the difference of the measured and the simulated downstream sound power level was $1.7 \mathrm{~dB}$. Totally the measured low frequency in duct sound power level downstream was $155.6 \mathrm{~dB}$ and simulated $156 \mathrm{~dB}$. As a conclusion the simulated acoustic source data can be considered quite accurate.

\section{Conclusions}

The goal of the present study was to investigate the medium speed IC-engine acoustic source characteristics numerically and experimentally not only in the low frequency - plane wave range but also in the high frequency range.

In this study the possibility to extract the acoustic source data for an exhaust system of a medium speed IC-engine using one-dimensional process simulation software has been tested. It was shown that the low frequency - plane-wave range induct exhaust noise of a medium speed IC-engine can be predicted reasonable accurately by using the GT-Power software. To predict the high frequency range noise, some other methods than the one-dimensional process simulation used in this study must be developed.

The high frequency range was included in the experimental acoustic power estimation using spatial averaging methods. The source data was estimated by averaging the measured auto- and cross-spectra and assuming negligible reflections and a semi-diffuse field. The sound power levels were higher when using the measured acoustic pressure auto spectra than the levels when using the measured acoustic pressure cross spectra. Estimating the downstream acoustic power by using the measured acoustic pressure cross spectra gave fairly similar results as the sound power estimation based on wave decomposition at the main engine cycle harmonics. According to this study, using the simple cross spectra averaging method instead of two microphone method to estimate the in-duct downstream acoustic power of medium speed IC-engine exhaust noise seems promising. To estimate the accuracy of the averaging methods used in the high frequency range, more experimental and numerical studies are necessary.

\section{REFERENCES}

1 M. L. Munjal. Acoustics of Ducts and Mufflers with Application to Exhaust and Ventilation System Design. John Wiley \& Sons, New York, 1987. 
2 D.E. Winterbone and R.J. Pearson. Design Techniques for Engine Manifolds, Wave Action Methods for IC-engines. Professional Engineering Publishing, 1999.

3 D.E. Winterbone and R.J. Pearson. Theory of Engine Manifold Design, Wave Action Methods for IC-engines. Professional Engineering Publishing, 2000.

4 M. L. Munjal. Acoustic characterization of an engine exhaust source - a review. Proceedings of Acoustics 2004, 2004.

5 F. Albertson, H. Bodén, and J. Gilbert. Comparison of different methods to couple nonlinear source descriptions in the time domain to linear system descriptions in the frequency domain - application to a simple valveless one-cylinder cold engine. Journal of Sound and Vibration, 291(3-5):963-985, 2006.

6 GT-POWER. User's Manual version 7.0. Gamma Technologies, 2009.

7 H. Bodén and M. Åbom. Modelling of fluid machines as sources of sound in duct and pipe systems. Acta Acustica, 3:1-12, Dec 1995.

8 V.H. Gupta and M. L. Munjal. On numerical prediction of the source characteristics on an engine exhaust system. Journal of the Acoustical Society of America, 92(5):2716-2725, 1992.

9 H. Bodén. On multi-load methods for determination of the source data of acoustic one-port sources. Journal of Sound and Vibration, 180(5):725-743, 1995.

${ }^{10}$ H. Bodén, A. Torregrosa, F. Olliver, K. Peat, R. Fairbrother, B. Henriksson, P. Recouvreur, O. Poullard, R. Glav, and J. Lavrentjev. Noise from turbo-charged diesel engine exhaust systems. Proceedings of the $12^{\text {th }}$ International Congress on Sound and Vibration, 2005.

11 R. Fairbrother, H. Bodén, and R. Glav. Linear acoustic exhaust system simulation using source data from non linear simulation. Proceedings of the 2005 SAE Noise and Vibration Conference, 2005.

12 H. Bodén. Recent advances in IC-engine acoustic source characterisation. Proceedings of the $14^{\text {th }}$ International Congress on Sound and Vibration, 2007.

13 M. Knutsson, H. Bodén, and J. Lennblad. On extraction of IC-engine intake acoustic source data from non-linear simulations. Proceedings of the $12^{\text {th }}$ International Congress on Sound and Vibration, 2005.

14 M. Knutsson. Modelling of IC-engine intake noise. Doctoral Thesis, The Royal Institute of Technology, Stockholm, Sweden, TRITA-AVE 2009:16, 2009.

15 W. Neise, W. Frommhold, F.P. Mechel, and F. Holste. Sound power determination in rectangular flow ducts. Journal of Sound and Vibration, 174(2):201-237, 1993.

16 J.Y. Chung and D.A. Blaser. Transfer function method of measuring in-duct acoustic properties. I-theory. II-experiment. Journal of the Acoustical Society of America, 68:907-921, Sep 1980.

17 H. Bodén and M. Åbom. Influence of errors on the two-microphone method for measuring acoustic properties in ducts. Journal of the Acoustical Society of America, 79(2):541-549, 1986.

18 P. Joseph, C.L. Morfey, and C.R. Lowis. Multi-mode sound transmission in ducts with flow. Journal of Sound and Vibration, 264(3):523-544, 2003. 